\title{
La traduction des régionalismes dans les nouvelles de Phanishwarnath Renu
}

Résumé: Toutes les œuvres littéraires sont représentatives de la langue et de la culture dont elles sont issues. De fait, notre identité culturelle est façonnée par la langue que nous parlons. Il en résulte que la traduction littéraire ne traite pas et ne peut pas traiter uniquement du transfert de la culture source dans la culture cible. Elle crée plutôt une identité de la culture source dans la culture cible. Cependant, au cours de ce processus de formation identitaire d'une culture particulière dans une culture étrangère à travers un texte littéraire, certains aspects sont ignorés, surtout si le texte source provient d'un pays multilingue - et donc multiculturel - comme l'Inde. La tentative de projeter l'idée d'unité dans la diversité fait perdre leur identité et leur unicité aux littératures régionales écrites dans les principales langues dominantes comme le hindi. Cet article tente de préserver cette identité régionale dans l'œuvre de Phanishwarnath Renu, écrite en hindi, dans sa traduction en français, en proposant la traduction avec des commentaires de certains passages présentant explicitement les concepts régionaux tels que chants, rites, cultes et croyances.

Mots clés: Traduction littéraire, régionalismes, culture, identité, sociolinguistique, littérature nationale, signes sociaux

\section{Introduction}

On ne peut pas isoler la langue de la culture et toute œuvre littéraire représente la langue ainsi que la culture qui est y enracinée. La traduction littéraire, à cette fin, ne reste plus un simple transfert du message et de l'histoire d'une langue à l'autre. Elle sert plutôt d'étude de deux cultures : la culture source et la culture cible, en étant un effort conscient pour connaître les deux cultures (Dingwaney et Maier 1995). Pierre Guiraud décrit les référents culturels comme des signes sociaux qui englobent les signes d'identité, les signes de politesse, les rites, les modes et les jeux. Les signes d'identité servent à reconnaître l'identité des individus et des groupes. Ces signes marquent l'appartenance d'un individu à un groupe social ou à une fonction, tandis que les signes de politesse marquent la nature des relations entre les individus. Par ailleurs, les rites sont des moyens de communication des groupes. Et les modes sont des manières d'être propres

Ә Open Access. () 2020 Runjhun Verma, published by De Gruyter. (c) BY-NC-ND This work is licensed under a Creative Commons Attribution-NonCommercial-NoDerivatives 4.0 License. https://doi.org/10.1515/9783110641998-013 
au groupe, alors que les jeux sont des imitations de la réalité sociale (Zhang 1999).

Chaque culture a son propre système de signes qui marque des valeurs sociales enracinées dans cette culture. Et ce sont ces valeurs et ces signes sociaux que représente une œuvre issue de cette culture. Souvent, quand on traduit les œuvres régionales dans une langue étrangère, les référents de la culture régionale se perdent totalement ; ce qui prive de fait le texte original de ses effets régionaux. L'idéologie nationaliste ne se concentre ni sur les attentes régionales, ni sur les divisions religieuses, ni sur les identités linguistiques (Kamala et Prasad 1997).

Depuis ces trois dernières décennies, la dimension culturelle dans la traductologie nous a obligée à lire et à analyser la traduction d'un texte littéraire non seulement au niveau du sens mais aussi au niveau de la culture et de sa représentation non manipulée. Cet article vise à traduire et à commenter certains exemples de régionalismes dans les nouvelles de Phanishwarnath Renu - avant tout Teesri Qasam -, un auteur connu pour avoir écrit dans un style régional.

\section{L'univers régional chez Phanishwarnath Renu}

Toute grande prose entretient des rapports étroits avec des langues régionales au sein d'un pays. Il est à noter que le terme « culture » est très vaste. On trouve plusieurs autres « sous-cultures », qui existent au sein de ladite grande culture d'un pays. Ici, la culture indienne est un mélange de plusieurs cultures régionales qui s’y trouvent et qui sont représentées dans la littérature de la région donnée. Le mot « régional », dans le contexte littéraire, comprend la prose ou bien la poésie qui dépeignent le paysage, le dialecte, les coutumes et le folklore propres à une région géographique. Les œuvres régionales évoquent donc plusieurs décors relatifs à la vie des habitants de la région. Elles expriment un lien étroit entre le lieu et les personnages. Elles essayent d'englober les rôles traditionnels, ethniques et socioéconomiques.

Phanishwarnath Renu est le premier écrivain régional dans la littérature hindi. Ses œuvres présentent une image unique du Bihar ${ }^{1}$ avec toutes ses nuances culturelles, linguistiques et sociohistoriques. Son premier ouvrage, Maila Anchal, a été signalé comme la première des œuvres régionales en Inde. Il a introduit un genre tout nouveau dans la littérature hindi, celui du régionalisme, où les aspects régionaux entrent dans la langue et la littérature dominante. Le régionalisme de

1 Région située dans l'Est de l'Inde. Phanishwarnath Renu a écrit des nouvelles et des romans dépeignant la vie rurale de cette région, d'où le concept du régionalisme dans ses œuvres. 
Renu, dans la littérature hindi, expose les caractéristiques exceptionnelles et propres à la région sans utiliser le dialecte, l'angik, de cette région.

Mais pourquoi les œuvres de Renu sont-elles qualifiées de « régionales » si elles ne sont pas écrites dans le dialecte propre à la région? Il expérimente avec l'univers oral des traditions dans ses œuvres. Il utilise les sons dominants chez ses personnages. Les mots sont transcrits par Renu tel qu'ils sont prononcés ou mal prononcés (dans le cas des mots anglais déformés) par les personnages et ainsi, il reproduit la qualité naïve du discours rural au Bihar, sans vraiment utiliser la langue et le dialecte régional. Le statut social et culturel des personnages ruraux se manifeste bien dans l'écriture de Renu (Hansen 1981).

Renu situe ses histoires dans les villages en pleine transformation dans une Inde devenue récemment indépendante. Il profite de son expérience de la vie dans un village pour esquisser les personnages de ses œuvres. Pour renforcer cette authenticité, il utilise une langue simplifiée, qui n'est pas uniquement le sanscrit, la langue des savants, ni le dialecte régional, l'angik, ce qui aurait pu rendre le texte inintelligible pour la plupart des lecteurs. Au contraire, ses œuvres sont écrites dans une sorte de mélange des langues que l'on peut appeler le hindoustani, langue fortement mélangée et simplifiée qui est parlée par la majorité de la population du Nord de l'Inde. Ce mélange de registres avec la langue soutenue, le dialecte et les mots anglais déformés représente la vie quotidienne des personnes qui ne sont pas allées à l'école et n'ont jamais eu contact avec un locuteur moderne et/ou urbain (Hansen 1981). Un autre aspect très intéressant qui renforce les spécificités des histoires dans les nouvelles de Renu est leur musicalité. Il profite de sa riche connaissance des traditions folkloriques en introduisant des chansons folkloriques au sein de ses œuvres. Ces chansons approfondissent la description de la pensée des personnages.

La nouvelle Maare Gaye Gulfam, écrite en 1954, a été adaptée au cinéma en 1966 dans le film intitulé Teesri Qasam. Cette nouvelle raconte l'histoire d'un villageois simple, Hiraman, qui fait trois vœux dans la vie : ne jamais faire entrer ou sortir les biens en contrebande, ne jamais faire transporter le bambou et ne jamais faire monter dans son char à bœufs une femme appartenant à une troupe de théâtre. L'histoire porte sur les circonstances et la situation qui obligent Hiraman à faire ce troisième vœu. Cette nouvelle se déroule dans le Nord du Bihar, à Farbisganj. Cependant, la première partie de l'histoire se passe dans le char à bœufs qui traverse différents villages afin d'arriver à Farbisganj. 


\section{L'importance de traduire les régionalismes pour un lecteur étranger}

Depuis les années 1980, l'aspect culturel dans la traduction et le discours postcolonial ont mis l'accent sur les aspects socioculturels des pays autrefois colonisés, en essayant de réaffirmer leur identité culturelle.

Ce travail se penche plutôt sur la traduction des aspects socioculturels régionaux, en distinguant la culture régionale du concept général de la culture indienne. On cherche à rendre en français les effets régionaux qui se trouvent chez Phanishwarnath Renu et ainsi, à accorder de l'importance à cette culture régionale. Notre objectif est aussi d'analyser la traduction de ces aspects socioculturels ainsi que sociolinguistiques régionaux en français, langue très différente en termes de champs sémantique, syntaxique et phonologique. On verra donc dans quelle mesure chacun de ces niveaux est traduisible. Il est à noter que la traduisibilité de ces éléments dépend aussi de la proximité des deux langues, la langue source et la langue cible.

L'aspect sociolinguistique attire ici notre attention sur le contexte social du vocabulaire d'un peuple. On y trouve les variantes des mots à l'intérieur d'une culture. Dans la traduction de cette nouvelle, on rencontre le problème de l'effacement ou bien l'unification de ces éléments hétérogènes qui pose une grande menace sur le caractère de cette nouvelle.

Dans de telles situations, la traduction des œuvres littéraires postcoloniales est utilisée comme un moyen de renforcer notre nouvelle identité sans stéréotypes. Elle devient un acte de résistance pour réparer les inanités et les injustices du passé (Bandia 2009). Notre but, dans cette étude, est d'amener les lecteurs vers le texte et l'idée originale de l'écrivain et non le contraire. Et pour ce faire, on propose de retenir les différences régionales dans la traduction pour que les lecteurs comprennent que l'Inde est un pays vaste et divers en matière de langues et de cultures. Pour préserver les régionalismes, il faut une approche sourcière. On essaye dès lors d'examiner la traduction comme un acte de réécriture et de reconstruction de l'identité culturelle. Cette nouvelle régionale où se trouve l'usage de dialectes, de sociolectes ou de néologismes, issus du pays autrefois colonisé, nous présente une occasion de traduire l'hétérogénéité linguistique au sein d'une communauté linguistique.

Les effets du régionalisme sont visibles dans les chansons folkloriques, les plats, les mœurs et les modes typiques de cette région. Pour montrer ces effets ici, on propose une grande catégorie de traduction : celle de la traduction des aspects sociolinguistiques, divisée encore en différents aspects sociaux. À l'aide de ces catégories, on essaiera d'éclairer les régionalismes, d'abord au niveau de la langue, puis au niveau des spécificités culturelles. 


\section{Les aspects sociolinguistiques}

La sociolinguistique actuelle attire notre attention sur le contexte social du vocabulaire d'un peuple. Il y a plusieurs variantes du mot à l'intérieur d'une culture : il existe, au sein du groupe même, des variations d'un individu à l'autre. Les variantes linguistiques se manifestent principalement sur trois plans : phonologique, grammatical et lexical (Zhang, 1999).

On suivra ce plan ici pour classifier l'usage des effets régionaux par l'écrivain à travers la langue.

\subsection{Phonologie}

Renu, dans ces deux nouvelles, montre les traditions orales de la langue. L'usage des sons y est très frappant.

Kacchhi sadak par ek chhote se khadd mein gaari ka dahina pahiya bemauke hichkola kha gaya. Hiraman ki gaari se halki sis ki awaz aayi. (Renu 2003, 434)

[Sur la route de campagne en terre battue, la roue de droite est tombée dans un petit trou. Le char a fait un léger son : sis $^{2}$.]

Dhan-dhan-dhan-dharam. Parda uth gaya. (Renu 2003, 450)

[Dhan-dhan-dhan-dharam. Le rideau a été tiré.]

On voit que Renu transpose les sons, tels quels, dans ses textes. Dans les deux exemples, on trouve que le son émis par les objets, se rattache au contexte culturel des personnages. Le son «sis » qui se fait entendre et que Renu transmet de façon onomatopéique nous rappelle cette région. On a gardé le même son dans le premier exemple afin de faire écho au texte original où Renu, au lieu d'éviter de tels sons et d'exprimer les sentiments dans la langue soutenue, préfère faire entrer l'oralité de la langue courante dans l'écrit. Le deuxième exemple nous indique le style unique de l'écrivain qui, au lieu d'écrire « au son du tambour, le rideau a été tiré », recourt à la translittération du son de tambour Dhan-dhan-dhan-dharam. C'est un exemple classique de l'oralité dans l'écriture de Renu.

2 La traduction de tous les extraits des œuvres de Renu est la nôtre. 


\title{
4.2 Lexique
}

Dans les œuvres de Renu, on trouve les variantes lexicales qui nous montrent les différences de classes et de castes. Cette nouvelle comprend des mots anglais mal prononcés par les personnages, dénotant ainsi leur manque d'éducation, mais aussi leur combat pour la modernité.

\begin{abstract}
Mathuramohan nautanki company main laila banane vali Hirabai ka naam kisne nahin suna hoga bhala. Lekin Hiraman ki baat nirali hai. Usne saat saal tak lagatar melon mein ladni laadi hai lekin kabhi nautanki theater nahin dekha. (Renu 2003, 435)

[Qui ne connaissait pas Hirabai, celle-là qui a joué le rôle de Laila dans la troupe de théâtre Mathuramohan? Mais Hiraman était différent, unique même. Pendant sept ans, il a transporté des biens à l'occasion des fêtes du village, mais il n'a jamais vu de pièce de théâtre ou de cinéma bioscope.]
\end{abstract}

Baksa dhone vale ne nautanki ke joker jaisa munh banakar kaha, "latfaram se bahar bhago. Bina ticket ke pakrega toh teen mahine ki hawa.” (Renu 2003, p. 457)

[Le porteur de bagage a dit, en grimaçant, à Hiraman : «Sors du quai. Si on te trouve sans billet, tu seras condamné à trois mois de prison. »]

Ici, on trouve l'usage de mots anglais déformés ou bien mal prononcés pour parler des choses qui sont nouvelles et modernes pour les villageois : par exemple, des mots comme latfaram qui est, en fait, le mot anglais platform, et dans nautanki theater company, on voit que le mot nautanki est ajouté à theater company, qui évoque le langage courant de la région dont il s'agit. Ces mots montrent l'arrivée du train et du théâtre ou bien du cinéma dans les villages indiens. Or, les gens ne parviennent pas à bien prononcer ces nouveaux mots qui nous ont été légués par les anciens colonisateurs. L'écrivain a gardé les prononciations incorrectes dans l'original pour décrire les aspects sociaux.

Cependant, dans la traduction de ces mots, on ne peut pas toujours garder les mêmes translittérations que dans l'original dans les cas des mots anglais. En effet, le but de leur utilisation dans les textes originaux est de marquer l'avènement de la modernité dans les villages indiens à travers la langue anglaise. Il est difficile de recréer cette fonction de la langue anglaise lorsqu'on traduit le texte en français, parce qu'il n'existe pas d'équivalents ou de structure pareille qui le permette.

\subsection{Les signes des mœurs}

La représentation correcte des mœurs dans la traduction est très importante. Les mœurs constituent, dans toute civilisation, une partie intégrante du patrimoine 
culturel. Elles contribuent à maintenir les relations entre les individus et l'ordre social. Elles ordonnent la vie sociale et elles deviennent, au cours du temps, les règles de savoir-vivre et de savoir-faire. L'identification de certaines idées avec certaines images est enracinée dans notre pensée. Par exemple :

\footnotetext{
Hiraman ki bhi shaadi hui thi, bachpan mein hi gaune ke pehle hi dulhin mar gayi. Hiraman ko apni dulhin ka chehra yaad nahi... doosri shaadi? [...] Bhabhi ki zidd, kumari ladki se hi karvayegi. Kumari ka matlab hai paanch-saat saal ki ladki. (Renu 2003, p. 436)

[Hiraman s'était marié, lui aussi, sa femme est morte quand elle était très jeune, avant même le gauna, moment où elle viendrait vivre sous le toit conjugal. Hiraman ne se souvient plus du visage de sa femme... Un second mariage ? [...] La belle-sœur insistait pour qu'il se marie à une jeune fille. Jeune veut dire une fille de cinq ou sept ans.]
}

Ici, cet exemple nous présente une image de la façon dont les personnages perçoivent la société et leur situation sociohistorique et socioéconomique, respectivement. On trouve une référence au mariage des enfants, pratique assez répandue aujourd'hui encore dans cette région. Dans certains villages indiens, à l'époque, la jeune mariée, en fait encore une fillette, reste chez ses parents jusqu'à la puberté avant d'aller chez son mari. Ce départ chez son mari est appelé le gauna. Il nous faut donc expliquer, en tant que traductrice, ce que signifie le mot gauna à l'aide de notes en bas de page ou d'un glossaire à la fin. On ne peut ni l'effacer, ni le remplacer par autre chose.

\subsection{Les faits religieux et les superstitions}

La religion occupe une place très élevée dans la société indienne. La vie quotidienne est très influencée par les cultes religieux, les rites, et ainsi de suite. Chaque action de la vie est déterminée par les faits religieux. La traduction de ces rites et de ces cérémonies pose un grand défi parce que ce sont, en quelque sorte, les moyens de communication des groupes.

Samne Champanagar se Sindhiya gaoan tak pahila hua maidan... kahin daakin pishachin toh nahin? (Renu 2009, p. 435)

[Devant, étendu, c'est le vaste terrain qui s'étale du village de Champanagar au village de Sindhya... Serait-ce une daakin pishachin ?]

Uski gaadi mein phir champa ka phool khila. Uss phool mein ek pari baithi hai... Jai Bhagwati! (Renu 2003, p. 436)

[Son char sentait encore l'odeur de frangipane. Et une fée assise sur cette fleur... Jai Bhagwati.] 
Ces deux exemples nous montrent la place que tient la religion chez les personnages dans les villages. Les gens remercient les Dieux pour les choses aussi simples que la présence d'une belle femme devant eux, par des expressions telles que Jai Bhagwati, qui signifie que c'est la déesse elle-même qui est apparue ici sous les traits de Hirabai. Les cultes religieux, les cérémonies, les rites, notamment funéraires, correspondent donc aux expériences et aux pratiques sociales des gens. Ces signes sociaux sont fortement connotés, exprimant la force, le pouvoir, l'aspiration ou l'humilité, et ces valeurs relèvent de la symbolique enracinée dans l'inconscient collectif (Zhang 1999).

On a ainsi essayé de garder ces aspects culturels par le biais d'emprunts, en expliquant le sens. Le premier exemple, daakin pishachin, montre la croyance surnaturelle dans l'inconscient collectif rural. Sa traduction en français entraînerait l'effacement de son sens, ce qui nous oblige à garder le mot tel quel, le concept de daakin pishachin étant propre à la culture indienne. Le premier mot, daakin, désigne la forme féminine d'un esprit malfaisant, tandis que le deuxième ne signifie pas uniquement l'esprit malfaisant, mais se rattache à la mythologie indienne où l'on trouve les démons appelés pishach et ainsi, la forme féminine pishachin. Dans le langage parlé, on utilise les deux mots ensemble parce que les deux concepts semblent se rattacher à quelque chose de surnaturel.

\subsection{Les signes idiomatiques}

Les signes idiomatiques marquent les caractères socioculturels de la langue sous forme d'expressions idiomatiques, de proverbes, de locutions, utilisés dans une œuvre littéraire. Les chansons folkloriques sont omniprésentes dans les œuvres de Renu. Ces chansons sont les marqueurs d'une histoire commune partagée par les membres d'une communauté donnée. C'est une tâche très difficile de traduire ces chansons avec tout leur lyrisme et les référents culturels.

\section{Laali-laali doliya mein}

Laali re dulhaniya

Paan khaye...

[Rouge est le doli, rouge est la nouvelle mariée, et elle mange du paan rouge...]

Dans cette chanson, la couleur rouge est dominante. Cette couleur a plusieurs significations ici : tout d'abord, le concept de doli est très indien ; c'est une sorte de palanquin, transporté par quatre personnes, dans lequel la nouvelle mariée arrive chez son mari. De plus, dans le Nord de l'Inde, la couleur rouge symbolise le bonheur conjugal. En outre, cette couleur connote également la gêne, puisque la mariée rougit parce qu'elle va rencontrer son mari et commencer une nouvelle vie 
avec lui. Enfin ici, dans cette chanson, elle mange du paan, le bétel, qui donne une couleur rouge à la bouche quand il est chiqué. Selon nous, il est important de traduire les chansons folkloriques littéralement, parce que leurs équivalents culturels n'existent pas dans la langue cible. D'ailleurs, le contexte où l'écrivain les a utilisées est aussi très important. Les chansons folkloriques jouent un rôle majeur dans la prose narrative.

\section{Conclusion}

Depuis quelques années, le nombre d'œuvres d'auteurs indiens traduites en français a augmenté. Les œuvres écrites en anglais, une des langues officielles du pays, par Salman Rushdie, Amitav Ghosh, Arundhati Roy et d'autres écrivains ont sûrement rendu l'Inde plus visible sur la scène littéraire française. Cependant, ces œuvres ne représentent pas entièrement le génie multilingue et multiculturel de l'Inde, par ailleurs si bien ancré dans les œuvres régionales ou bien les littératures bhasha. Il existe plusieurs langues et dialectes au sein du pays, et il y a un fort échange entre les littératures issues de ces langues. Mais observe-t-on la présence de ces littératures au niveau international ? La plupart des œuvres présentes sur la scène internationale sont rédigées en anglais ou traduites en anglais, la langue qui fait parfois office de filtre pour des littératures régionales. Nicola Pozza constate que dans de telles situations, il existe un modèle binaire des traductions et des cultures qui est souvent renforcé par la hiérarchie entre les langues dominées/minoritaires et les langues dominantes (Pozza 2010). Les bhasha ou langues régionales, y compris le hindi, possèdent un statut minoritaire en ce qui concerne les traductions faites depuis l'Inde.

À travers le cadre théorique et certains exemples de la traduction de la nouvelle Teesri Qasam, on a essayé d'illustrer la traduction du caractère régional dans les œuvres de Renu, du hindi vers le français. L'objectif était de montrer la présence des diverses cultures qui existent au sein d'une grande culture indienne, de renforcer l'identité de ces cultures, qui est celle du Bihar dans notre cas, et de rendre les œuvres des langues régionales plus visibles sur la scène littéraire internationale. Dans les exemples présentés ici, on a adopté une approche plutôt sourcière, afin de présenter les mêmes complexités langagières qui caractérisent les nouvelles originales. Les œuvres de Renu ne sont pas faciles à traduire à cause de leur caractère polyphonique et polysémique et ainsi, en tant que traducteur, on doit être très conscient de représenter l'étrangeté de ces œuvres comme elle se manifeste même en hindi. Il est nécessaire d'expliquer les différences et d'inviter les lecteurs à découvrir une nouvelle culture et à l'apprécier, au lieu de tout effacer et/ou assimiler en se soumettant, de nouveau, à l’impérialisme culturel. 


\section{Bibliographie}

Bassnett, Susan, et Harish Trivedi. Post Colonial Translation. Theory and Practice. Londres : Routledge, 1999.

Berman, Antoine. L'épreuve de l'étranger. Paris : Gallimard, 1984.

Berman, Antoine, et Gérard Granel, Annick Jaulin, Georges Mailhos, Henri Meschonnic, et al.

Les Tours de Babel. Essais sur la Traduction. Mauvezin : Trans-Europ-Repress, 1985.

Burger, Maya, et Nicola Pozza (dir.). Translation Through Hindi Literature: A Plurality of Voices.

Berne : Peter Lang, 2010.

Chaudhari, Surendra. Phanishwar Nath Renu. New Delhi : Sahitya Akademi, 2000.

Dingwaney, Anuradha, et Carol Maier. Between Languages and Cultures. Delhi : Oxford University Press, 1991.

Hansen, Kathryn. « Renu's regionalism: Language and Form ». The Journal of Asian Studies 40.2 (1981) : 273-294.

Kumar, Suvas (éd.). Phanishwar Nath Renu Sanchayita. Delhi : Medha Books, 2003.

Ladmiral, Jean-René. "Sourciers et ciblistes ». La Traduction. Revue d'esthétique 12 (1986) : 33-42.

Mukherjee, Arun. Oppositional Aesthetics. Toronto : TSAR Publications, 1994.

Kamala, N., et G. J. V. Prasad. «Transcreating India(s): The Nation in English Translation ». META 42.2 (1997) : 445-455.

Milton, John et Paul Bandia (éds), Agents of Translation, Amsterdam/ Philadelphia: John Benjamins Publishing Company, 2009.

Pozza, Nicola. «Translating from India and the Moving Space of Translation ». India in Translation Through Hindi Literature: A Plurality of Voices. Maya Burger et Nicola Pozza (dir.).

Berne : Peter Lang, 2010. 127-152.

Pushp, Robin Shaw. Sone ki kalam wala Hiraman. Ghaziabad : Ankur Prakashan, 1981.

Renu, Phanishwarnath. « Mare Gaye Gulfam ». Thumari. New Delhi : Rajkamal Publications, 2009.

Renu, Phanishwarnath. Phanishwarnath Renu Sanchayita. Kumar, Suvas (dir.). New Delhi : Medha Books, 2003.

Shephalika. Renu ka katha-sansar. New Delhi : RadhaKrishna Prakashan, 1996.

Zhang, Xinmu. « Les signes sociaux et leur traduction ». META 44.1 (1999) : 105-112.

Dr. Runjhun Verma is a teacher of French at the Delhi School of Journalism, University of Delhi and at Alliance française de Delhi. She has a keen interest in literary translation and intercultural studies. Her doctoral thesis dealt with the politics of translation of Indian literary works into French. 